\title{
Study on Tribological Behavior Based on 2D Zn- MOFs Interface Enrichment of Oil-In-Water Emulsion
}

\author{
Fei-Fei Wang \\ Yangzhou University \\ Haiyang Ding \\ Yangzhou University \\ Guang Tan \\ Yangzhou University \\ Zan Liu \\ Yangzhou University \\ zhilin cheng ( $\nabla$ zlcheng224@126.com ) \\ Yangzhou University
}

\section{Research Article}

Keywords: 2D MOFs, Oil-in water emulsion, Emulsifying property, Tribological behavior

Posted Date: February 15th, 2021

DOl: https://doi.org/10.21203/rs.3.rs-176720/v1

License: (c) (1) This work is licensed under a Creative Commons Attribution 4.0 International License. Read Full License 


\section{Abstract}

For the first time, we attempt to use two-dimensional Zn-based metal organic framework (2D Zn-MOFs) nanosheets as emulsifiers and additives in oil-in-water emulsions, including 2D Zn(Bim)(OAc) (1) nanosheets, 2D Zn(Bim)(OAc) (2) nanosheets and 2D ZnBDC nanosheets. The emulsification results showed that the prepared emulsion was uniform and stable, and 2D Zn(Bim)(OAc) (1) emulsion has the most significant performance in terms of anti-friction and anti-wear performance and improvement of extreme pressure performance. Compared with the base emulsion, the friction coefficient and wear volume were reduced by $22 \%$ and $53.6 \%$, respectively, while the last non-seizure load $\left(P_{B}\right)$ was increased by $15.5 \%$. Finally, the friction mechanism was discussed and proposed.

\section{Introduction}

Water based metalworking fluid has been widely used in metal and alloy cutting, stamping, rolling, drawing and other processing fields due to its advantages of good cooling performance, low cost, environmental protection, low toxicity and fire resistance, and has the potential to gradually replace oilbased lubricants $[1,2]$. However, the water-based metalworking fluid has the characteristics of low viscosity and low coefficient of viscosity and pressure because it contains a lot of water, which makes it difficult to form an effective lubricating film in the friction process and has the defect of poor lubricity. The addition of functional additives was one of the effective means to enhance the tribological properties of water-based fluids. The functional additives could reacted with the friction surface to form a lubricating film during the friction process $[3,4]$.

In recent years, two-dimensional (2D) nanomaterials has been considered as an ideal alternative to traditional extreme pressure (EP) and anti-wear (AW) additives due to their unique interlayer structure and excellent tribological properties [5-8]. For example, the friction coefficient and wear volume of the base oil decreased by $49.4 \%$ and $90 \%$ respectively when $\mathrm{MoS}_{2}$ nanosheets were used as base oil additives [9]; when graphene oxide nanosheets were used as ionic liquid additives, the friction coefficient and wear volume of the base oil decreased by 600 The results shown that the ionic liquid reaches the liquid super lubrication state at the extreme pressure of $0.005 \mathrm{MPa}$ [10]; when graphene oxide nanosheets were used as additives for glycol aqueous solution, the aqueous solution also reaches the super lubrication state [11]. However, due to the aggregation or deposition of two-dimensional inorganic nanosheets and the poor natural interface with organic molecules, their practical application in lubricants was seriously limited. Recently, researchers has discovered that the modified two-dimensional nanomaterials were amphiphilic nanomaterials, which could be used as solid emulsifiers to reduce the interfacial tension of oil and water to form Pickering emulsions. The application of Pickering emulsions to metalworking fluids could effectively solve interface science problems $[12,13]$. For example, functionalized graphene oxide nanosheets has good dispersibility, load-carrying capacity, anti-friction and anti-wear properties in an oilin-water emulsion system [14-17]. However, surface modification technology not only slightly increased the viscosity of oil dispersion, but also makes it impossible to realize process up scaling [18]. 
As we all know, metal organic frameworks (MOFs) have been widely used in adsorption, separation and catalysis due to their large specific surface area, controllable morphology and functionalization of metal ions and organic ligands [19]. Therefore, two-dimensional metal organic frameworks (2D MOFs) nanomaterials could also be used as an effective substitute for functional additives to improve the tribological properties of water-based metalworking fluids. In previous work, we explored the tribological properties of 2D MOFs as lubricating oil additives. The research results shown that the friction reducing and anti-wear properties of 2D MOFs in lubricating oil were not as good as those of 2D inorganic nanomaterials, but it owned good dispersion properties due to their excellent interface with organic solvents [20-22]. For example, 2D Zn (Bim) (OAc) nanosheets were translucent and stably dispersed in base oil for 3 days without modification, and their friction coefficient and wear volume as lubricant additives were reduced by $17 \%$ and $22.6 \%$, respectively [21]. Because 2D MOFs has two affinity properties to self-assemble at the oil-water interface, the interfacial tension was reduced, and the Pickering emulsion [23-25] stabilized by solid particles was formed. Therefore, 2D MOFs could be used as solid surfactants in metalworking fluids without modification, aiming to solved the stability problem of 2D MOFs nanomaterials and improved their tribological properties.

In this paper, 3 kinds of 2D Zn-MOFs nanosheets (2D Zn (Bim) (OAc) (1) nanosheets, 2D Zn (Bim) (OAc) (2) nanosheets and 2D ZnBDC nanosheets) were applied to oil-in-water emulsion to study the effect of additives on their friction properties. Through a series of characterizations, the forms of 2D Zn-MOFs nanosheets in emulsion and the stability of emulsions were studied. Finally, the lubricating properties and extreme pressure properties of the oil-in-water emulsion were studied by tribological experiments and extreme pressure tests. The lubrication mechanism of the emulsion was further analyzed by X-ray Photoelectron Spectroscopy (XPS).

\section{Experimental}

\subsection{Materials}

zinc nitrate hexahydrate $\left(\mathrm{Zn}\left(\mathrm{NO}_{3}\right)_{2} \cdot 6 \mathrm{H}_{2} \mathrm{O}, \mathrm{AR}, \geq 99.0 \%\right)$, Zinc acetate dehydrate $\left(\mathrm{Zn}\left(\mathrm{CH}_{3} \mathrm{COO}\right)_{2} \cdot 2 \mathrm{H}_{2} \mathrm{O}, \mathrm{AR}\right.$, $\geq 99.0 \%$ ), ethanol ( $E t O H, A R, 98 \%)$, methanol ( $M e O H, A R, 98 \%)$, dimethylformamide (DMF, $\geq 99.5 \%)$ ) were purchased from Sinopharm Chemical Reagent Co., Ltd. Benzimidazole (Bim, AR, 98\%) was supplied by Shanghai Energy Chemical Co., Ltd (Shanghai, China). Terephthalic acid $\left(\mathrm{H}_{2} \mathrm{BDC}, 99.0 \%\right)$ and tween-85 (TW85, 99\%) were provided by Aladdin Industrial Corporation (Shanghai, China). $150 \mathrm{SN}$ base oil $\left(40^{\circ} \mathrm{C}\right.$ kinematic viscosity of $28.8-35.2$, flash point of $214{ }^{\circ} \mathrm{C}$, pour point of $-9{ }^{\circ} \mathrm{C}$ ) was supplied by Shenzhen Lubricant Co., Ltd (China). Deionized water was made by Yangzhou University (Jiangsu, China).

\subsection{Preparation of 2D Zn-MOFs oil-in-water emulsion}

Firstly, 2D Zn (Bim) (OAc) (1) nanosheets, 2D Zn (Bim) (OAc) (2) nanosheets and 2D ZnBDC nanosheets were prepared by ultrasonic assisted stripping method, liquid / liquid interface method and solvent modulation method, respectively. Their preparation process and characterization were detailed in the 
previous work [20-22]. According to references [20-22], the molecular structures of 2D Zn (Bim) (OAc) (1) nanosheets, 2D Zn (Bim) (OAc) (2) nanosheets and 2D ZnBDC nanosheets were characterized by AFM, XRD, FT-IR, Raman, EDS, SEM and TEM. Here we only showed the SEM images (Fig. 1A-C) and the summary of other characteristics (transverse size, thickness and lattice spacing) (Table 1). Then the traditional surfactant TW85, base oil 150SN and deionized water were selected as the main materials of emulsifier. Tw85 $(0.75 \mathrm{~g})$ was swelled in the base oil $(0.45 \mathrm{~g})$, and 2D Zn-MOFs $(0.0375 \mathrm{~g})$ was added to form the oil phase. Then the oil phase was ultrasonically dispersed at $25^{\circ} \mathrm{C}$ for 30 minutes, and then magnetically stirred for 10 minutes. Then deionized water $(13.8 \mathrm{~g})$ was added to the oil phase and stirred for 30 minutes. 2D Zn (Bim) (OAc) (1) emulsion, 2D Zn (Bim) (OAc) (2) emulsion and 2D ZnBDC emulsion were obtained, respectively. The basic emulsion without 2D Zn-MOFs nanosheets was prepared as a comparison (Fig. 1D).

Table 1

The summary of characteristic information of 2D Zn-MOFs

\begin{tabular}{|c|c|c|c|}
\hline Sample & Lateral size & Thickness (nm) & lattice distance (nm) \\
\hline 2D Zn(Bim)(OAc) (1) nanosheets & \multirow{3}{*}{$\begin{array}{l}\text { Hundreds of } \\
\text { nanometers to } \\
\text { several microns }\end{array}$} & 4 & 1.08 \\
\hline 2D Zn(Bim)(OAc) (2) nanosheets & & 2.64 & 1.05 \\
\hline 2D ZnBDC nanosheets & & 5 & 1.28 \\
\hline
\end{tabular}

\subsection{Tribological properties of emulsion}

The friction and wear tests of the ball and slide reciprocating MFT-5000 type multi-function friction and wear tester (Atec, USA) were carried out. The upper part of the friction pair is 304 steel ball with diameter of $6 \mathrm{~mm}$, and the lower part was 304 steel slider with length of $30 \mathrm{~mm}$, width of $15 \mathrm{~mm}$ and height of $4 \mathrm{~mm}$. According to the actual rolling contact stress, the friction test conditions were load $30 \mathrm{~N}$, frequency $2 \mathrm{~Hz}$, reciprocating distance $1.5 \mathrm{~mm}$ and friction time $30 \mathrm{~min}$. Before the test, the friction pair was cleaned with the mixture of ethanol and petroleum ether, and the test was carried out after air drying. Each friction test was repeated at least three times. The extreme pressure $\left(P_{B}\right)$ value of the emulsion was tested at the rotational speed of $1200 \mathrm{rpm}$ by using four ball friction machine (MMW-1, Jinan Chenda Ltd Co., China).

After the friction and wear test, the worn surface morphologies and wear volume of the steel slider were also examined using the white light interference three-dimensional surface topography system and threedimensional measurement laser microscope (LSM700, Zeiss, Germany). The chemical composition on the worn surface was determined using XPS (ESCALAB 250Xi, Thermo, USA).

\subsection{Characterizations}

The morphology of 2D MOFs nanosheets was characterized using the scanning electron microscope (SEM, Zeiss-supra55, Germany) at an accelerating voltage of $10.0 \mathrm{kV}$. The viscosity were conducted using the rotational rheometer (RS600, Thervno, USA) under various shear speeds that ranged from 0.01$1000 \mathrm{~s}^{-1}$ for $120 \mathrm{~s}$ at $25{ }^{\circ} \mathrm{C}$. Micrograph of droplet distribution in emulsion were taken on professional 
polarizing microscope (DM2700P, Leica, Germany). Particle size distribution of oil droplets in emulsion was monitored by nanoparticle analyzer (Zen3690, Malvern, UK). The interface tension test of the emulsion was carried out with BEY3B automatic meter/interfacial tension meter.

\section{Results And Discussion}

Figure 2 shows the morphology of large droplets on zeroth days after preparation of base emulsion, 2D Zn (Bim) (OAc) (1) emulsion, 2D Zn (Bim) (OAc) (2) emulsion and 2D ZnBDC emulsion. It can be seen that the incorporation of 2D Zn-MOFs nanosheets into the base emulsion leads to a decrease in the droplet diameter and the presence of surfactants at some water oil interfaces. In contrast to Fig. B-D, it can be found that the probability of 2D Zn (Bim) (OAc) (2) nanosheets appearing at the water oil interface is even greater. $2 \mathrm{D} Z \mathrm{Zn}-\mathrm{MOF}$ nanosheets distributed in the emulsion droplet size are ranked as $2 \mathrm{D}$ $\mathrm{Zn}(\mathrm{Bim})(\mathrm{OAc})$ (1) emulsion < 2D ZnBDC emulsion <2D Zn(Bim)(OAc) (2) emulsion, which corresponds to the thickness of nanosheets in Table 1. It indicates that the emulsifying property of 2D Zn (Bim) (OAc) (1) emulsion with better thickness is better.

Figure $3 \mathrm{~A}$ shows the change of interfacial tension between water and oil in 2D Zn-MOFs nanosheets incorporated into the base emulsion. It is found that the interfacial tension of the base emulsion after the incorporation of 2D Zn-MOFs nanosheets decreases. The interfacial tension of 2D Zn (Bim) (OAC) (1) emulsion is at least $30.9 \mathrm{~N} \mathrm{~m} / \mathrm{m}$, which corresponds to the corresponding Fig. 2, indicating that $2 \mathrm{D} \mathrm{Zn}$ $(\mathrm{Bim})(\mathrm{OAC})(1)$ nanosheets have greater ability to regulate interfacial tension [26]. Figure $3 \mathrm{C}$ shows that on the 0 day after preparation, the average particle size of the base emulsion, 2D $\mathrm{Zn}$ (Bim) (OAc) (1) emulsion, 2D Zn (Bim) (OAc) (2) emulsion and 2D ZnBDC emulsion is about 813, 150, 900 and $820 \mathrm{~nm}$, respectively. The average particle size of $2 \mathrm{D} \mathrm{Zn} \mathrm{(Bim)} \mathrm{(OAc)} \mathrm{(2)} \mathrm{emulsion} \mathrm{is} \mathrm{smaller} \mathrm{than} \mathrm{that} \mathrm{of} \mathrm{other}$ emulsions, indicating that it is beneficial to the formation of smaller droplets with higher stability. In order to effectively reflect the stability of 2D Zn-MOFs series emulsion, the viscosity and droplet size changes with time are monitored in this paper, as shown in Fig. 3B, D. As shown in Fig. 3B, the viscosity of the emulsion changes little with time, indicating that its stability is good. As can be seen from Fig. 3D, the droplet size of emulsion drops slightly in all samples over time. When the emulsion was placed for 15 days, the particle size of the 2D Zn-MOFs series emulsion was smaller than that of the base emulsion, indicating that its stability was better than that of the base emulsion.

Figure 4 shows the tribological properties of base emulsion and 2D Zn-MOFs series emulsion at $30 \mathrm{~N}$ loading. Obviously, the friction coefficient of 2D Zn-MOFs series emulsion is lower than that of base emulsion. As shown in Fig. 4A, it shows that 2D Zn-MOFs series emulsion has better friction reducing performance than base emulsion. This may be attributed to the 2D Zn-MOFs nanosheets at the interface of the emulsion can flow into the trench through the flow of the emulsion, and then deposit in the groove to form a layer of lubrication protection film $[16,27]$. Compared with base emulsion (Fig. 4B), the average friction coefficient and wear volume of $2 \mathrm{D} \mathrm{Zn} \mathrm{(Bim)} \mathrm{(OAC)} \mathrm{(1)} \mathrm{emulsion} \mathrm{were} \mathrm{decreased} \mathrm{by} \mathrm{about} 22 \%$ and 53.6\%; 2D Zn (Bim) (OAc) (1) emulsion were decreased by about $17.3 \%$ and $38.7 \%$; $2 \mathrm{D} \mathrm{ZnBDC}$ emulsion were decreased by about $18 \%$ and $48 \%$, respectively. The results show that the friction property of $2 \mathrm{D} \mathrm{Zn}$ 
(Bim) (OAc) (1) emulsion is the best, which may be attributed to the smallest particle size and good stability of the emulsion droplet (Fig. 3), and the 2D Zn (Bim) (OAc) (1) nanosheets into the emulsion flow process are more, and the lubricant film formed is thicker[28]. Figure $4 \mathrm{C}$ shows the bearing capacity of base emulsion and 2D Zn-MOFs series emulsion. It can be clearly found that the PB value of 2D Zn-MOFs series emulsion is higher than that of base emulsion, indicating that the addition of 2D Zn-MOFs nanosheets can promote the extreme pressure of emulsion. In particular, the 2D $\mathrm{Zn}$ (Bim) (OAc) (1) emulsion with low viscosity and small particle size increased by $15.5 \%$ compared to the base emulsion PB value, indicating that 2D Zn (Bim) (OAc) (1) nanosheets had good loading capacity. This is attributed to the best dispersibility and stability of $\mathrm{Zn}(\mathrm{Bim})(\mathrm{OAc})(1)$ nanosheets in emulsion.

Figure 4D is a wear trace on the surface of 304 slider when lubricated by base emulsion and 2D Zn-MOFs emulsion when the reciprocating frequency is $2 \mathrm{~Hz}$, the load is $30 \mathrm{~N}$ and the time is $30 \mathrm{~min}$. The wear of the 304 slider on the lubrication of base emulsion and 2D Zn-MOFs emulsion (Fig. 5). According to Figs. $4 \mathrm{D}$ and 5 , the wear marks of the base emulsion are the largest and the deepest, the depth is about $5 \mu \mathrm{m}$, the wear width is about $411 \mu \mathrm{m}$. Secondly, the wear marks of 2D Zn (Bim) (OAc) (2) emulsion are slightly lower than the base emulsion, the depth is $4 \mu \mathrm{m}$ and the wear width is $368 \mu \mathrm{m}$; and the wear scar of $2 \mathrm{D} \mathrm{Zn} \mathrm{(Bim)} \mathrm{(OAc)} \mathrm{(1)} \mathrm{emulsion} \mathrm{is} \mathrm{the} \mathrm{lightest} \mathrm{and} \mathrm{smooth,} \mathrm{the} \mathrm{depth} \mathrm{is} 2.5 \mu \mathrm{m}$, and the wear width is about $341 \mu \mathrm{m}$. Thereafter, the statistical results of wear volume have a great relationship with wear morphology. The order of anti-wear property was 2D Zn (Bim) (OAC) (1) emulsion < 2D ZnBDC emulsion < 2D Zn (Bim) (OAc) (2) emulsion < base emulsion. This may be attributed to 2D Zn-MOFs nanosheets, which might create a solid lubrication film on the rubbing surface and effectively reduce friction and wear [13].

In order to find out the lubricating mechanism of 2D Zn-MOFs emulsion, XPS was used to determine the composition of the lubricant film on the surface of the slider, as shown in Fig. 6 . The results of elemental analysis of XPS showed that the friction film of the base emulsion on the 304 slider consisted of $64.5 \% \mathrm{C}$, $25.12 \% \mathrm{O}$ and $5.45 \% \mathrm{Fe}$, while the friction film formed by 2D Zn (Bim) (OAc) (1) emulsion was composed of $62.93 \% \mathrm{C}, 27.71 \% \mathrm{O}, 1.15 \% \mathrm{~N}, 27.71 \% \mathrm{O}$ and $2.73 \%$. The results show that $2 \mathrm{D} \mathrm{Zn}-\mathrm{MOFs}$ emulsion reduces friction by forming friction film at the friction interface. The results of Fig. $6 \mathrm{~B}$ show that the $\mathrm{C}$ element on the worn surface mainly exists in two forms: non oxidized carbon ring ( $\mathrm{C}-\mathrm{C} / \mathrm{C}=\mathrm{C}, \sim 285 \mathrm{EV}$ ) and hydroxyl group (C-OH, $284 \mathrm{EV}$ ) [12-14]. In these forms, $\mathrm{C}-\mathrm{C} / \mathrm{C}=\mathrm{C}$ and $\mathrm{C}-\mathrm{O}$ bonds are organic ligands corresponding to 2D Zn-MOFs or produced by friction process when fresh metal surfaces are exposed to emulsion and air. $\mathrm{O}$ exists in the form of iron oxide ( $\mathrm{FeO}, \mathrm{FeOOH}, \mathrm{Fe}_{2} \mathrm{O}_{3}, \sim 530 \mathrm{ev}$ ) [15]. $\mathrm{Fe}$ exists in the form of $\mathrm{Fe}(\sim 720 \mathrm{EV})$ and iron oxide (FeO, FeOOH, $710 \mathrm{EV})$ [14]. The presence of iron oxide, $\mathrm{C}-\mathrm{OH}$ and $\mathrm{Zn}$ indicates that the $2 \mathrm{D} \mathrm{Zn}-\mathrm{MOF}$ s emulsion has been reacted with the metal surface and formed a tribological film. The above results show that 2D Zn MOFs nanosheets act as boundary lubrication film and have the ability to protect the surface, thus showing better anti-wear ability.

In summary, during the friction process, 2D Zn-MOFs emulsion had the friction-reducing and anti-wear capacities. Based on the worn surface analysis, the tribological mechanism of 2D Zn-MOFs emulsion can be proposed as the following three aspects. First, 2D Zn-MOFs emulsion results in good lubricity due to 
its small droplets [28]. Therefore, compared with other emulsions, the 2D Zn(Bim)(OAc) (1) emulsion with a droplet size of $150 \mathrm{~nm}$ has more significant anti-friction and anti-wear properties. Second, 2D Zn-MOFs emulsion can form the adsorption film and tribo-film between the contact surfaces. After the breakup of 2D Zn-MOFs coated droplets, the flexible thin film structure of 2D Zn-MOFs nanosheets endows them easily be transferred into the contact surfaces, and further adsorbed and deposited on the friction surfaces to form an adsorption film. Moreover, through XPS analysis, it can be seen that some 2D ZnMOFs nanosheets had reacted with the rubbing surfaces, and a tribo-film had been formed. Hence, $2 \mathrm{D} \mathrm{Zn-}$ MOFs nanosheets can be bound more tightly with the metal surfaces through the formation of chemical bonds. Adsorption film and tribo-film may work together to reduce COF and wear of the contact surfaces.

\section{Conclusions}

In this study, 2D Zn-MOFs nanosheets were selected as emulsifiers and additives to be used in oil-in-water emulsions. 2D Zn-MOFs emulsion has good stability and tribological properties.

(1) The nanometer particle size distribution of the emulsion and the results of the optical microscope image showed that the prepared emulsion and 2D Zn-MOFs emulsion could be stable and uniform for more than half a month.

(2) The results of friction and extreme pressure tests showed that the tribological properties of three kinds of 2D Zn-MOFs emulsions showed similar trends, which could be summarized as $2 \mathrm{D} \mathrm{Zn}(\mathrm{Bim})(\mathrm{OAc})(1)$ emulsion > 2D ZnBDC emulsion > 2D Zn (Bim)(OAc) (2) emulsion > base emulsion. Compared with the base emulsion, the friction coefficient and wear volume of $2 \mathrm{D} \mathrm{Zn}(\mathrm{Bim})(\mathrm{OAc})(1)$ emulsion were reduced by $22 \%$ and $53.6 \%$, respectively, and the PB value was increased by $15.5 \%$.

(3)Tribological mechanism of 2D Zn-MOFs emulsions may be due to the following two aspects: the excellent lubricity of the small droplets in 2D Zn(Bim)(OAc) (1) emulsion; the formed adsorption film and tribo-film between the two rubbing surfaces. These two aspects may work together to reduce COF and wear of the contact surfaces.

\section{Declarations}

\section{Acknowledgment}

This work was funded by postgraduate research \& practice innovation program of Jiangsu province (XSJCX19-064) and the Jiangsu Provincial Colleges and Universities First-Class Project Program (PPZY2015B112). The data of this paper originated from the Test Center of Yangzhou University.

\section{References}

1. Osama, M., Singh, A., Walvekar, R.: Mohammad Khalid, Thummalapalli Chandra Sekhara Manikyam Gupta, Wong Wai Yin. Recent developments and performance review of metal working fluids. Tribol. 
Int. 114, 389-401 (2017)

2. Wickramasinghe, K.C., Sasahara, H., Rahim, E.A., Perera, G.I.P.: Green metalworking fluids for sustainable machining applications: A review. J. Clean. Prod. 257, 120552 (2020)

3. Jincan, Y., Zeng, X., Ren, T.: Emile van der Heide. Boundary lubrication of stainless steel and CoCrMo alloy based on phosphorous and boron compounds in oil-in-water emulsion. Appl. Surf. Sci. 315, 415-424 (2014)

4. Tomala, A., Karpinska, A., Werner, W.S.M., Olver, A.: H. Störi. Tribological properties of additives for water-based lubricants. Wear 269, 804-810 (2010)

5. Huaping, Xiao: Shuhai Liu. 2D nanomaterials as lubricant additive: A review. Mater. Des. 135, 319332 (2017)

6. Lu Ma, Z., Liu, Z., Cheng: Scalable exfoliation and friction performance of few-layered $\mathrm{WS}_{2}$ nanosheets by microwave-assisted liquid-phase sonication. Ceram. Int. 46, 3786-3792 (2020)

7. Zhilin Cheng, Z., Ma, H., Ding, Z., Liu: Environmentally friendly, scalable exfoliation for few-layered hexagonal boron nitride nanosheets (BNNSs) by multi-time thermal expansion based on released gases. Journal of Materials Chemistry C 7, 14701-14708 (2019)

8. Peirong Wu, W., Li, Z., Liu, Z., Cheng: Preparation and tribological properties of oleic acid-decorated $\mathrm{MoS}_{2}$ nanosheets with good oil dispersion. J. Dispersion Sci. Technol. 39, 1742-1751 (2018)

9. Yi, M., Zhang, C.: The synthesis of two-dimensional $\mathrm{MoS}_{2}$ nanosheets with enhanced tribological properties as oil additives. RSC Advances 8, 9564-9573 (2018)

10. Xiangyu Ge, J., Li, H., Wang, C., Zhang, Y., Liu, J., Luo: Macroscale superlubricity under extreme pressure enabled by the combination of graphene-oxide nanosheets with ionic liquid. Carbon 151, 76-83 (2019)

11. Xiangyu Ge, J., Li, R., Luo, C., Zhang, J., Luo: Macroscale superlubricity enabled by the synergy effect of graphene-oxide nanoflakes and ethanediol. ACS Appl. Mater. Interfaces. 10, 40863-40870 (2018)

12. Yang, H., Xue, S., Zhou, J., Li, J., Zeng, X.: Interfacial assembly behavior of alkylamine-modulated graphene oxide with different oxidation degrees. Langmuir 35, 12936-12946 (2019)

13. Yinglei Wu, X., Zeng, T., Ren: Erik de Vries, Emile van der Heide. The emulsifying and tribological properties of modified graphene oxide in oil-in-water emulsion. Tribol. Int. 105, 304-316 (2017)

14. Hongmei Yang, J., Li, X., Zeng: Tribological behavior of nanocarbon materials with different dimensions in aqueous systems. Friction 8, 29-46 (2018)

15. Yang, H., Zhao, L., Xue, S., Deng, Z., Li, J., Zeng, X.: Branch-chain length modulated graphene oxides for regulating the physicochemical and tribophysical properties of pickering emulsions. Colloids and Surfaces A: Physicochemical and Engineering Aspects 2019, 579

16. Shaoqing Xue, Y., Cen, H., Yang, T., Honda, Y., Nakanishi, L., Zhang, B., Zhang, Xiangqiong Zeng. The Enhanced Lubrication of Water-Based Cutting Fluid by Functionalized GO. Tribology Letters 2020, 68

17. Hongmei Yang, J., Li, X., Zeng: Triobological Behavior of Pickering Emulsions Stabilized with Alkylamine Edge-functionalized Graphene Oxide. China Surface Engineering 32, 151-159 (2019) 
18. Deepika: Nanotechnology implications for high performance lubricants. SN Applied Sciences 2, 1128 (2020)

19. Ko, M., Mendecki, L., Mirica, K.A.: Conductive two-dimensional metal-organic frameworks as multifunctional materials. Chem. Commun. 54, 7873-7891 (2018)

20. Wang, F., Liu, L.F.Z., Cheng, Z.: Surfactant-mediated preparation and tribological behaviors of fewlayer ZnBDC. Mater. Lett. 257, 126757 (2019)

21. Feifei Wang, Z., Liu, Z., Cheng. Ultrasonic-assisted exfoliation for 2D Zn(Bim)(OAc) nanosheets used as an oil-soluble additive in lubricants. Applied Organometallic Chemistry 2020, e5950

22. Wang, F.-F., Ding, H., Tan, G., Liu, Z., Cheng, Z.-L.: Interface synthesis for controllable construction of 2D Zn(Bim)(OAc) nanosheets via oil/water system and their application in oil. CrystEngComm 2021

23. Ping Song, G., Natale, J., Wang, T., Bond, H., Hejazi: Hector de la Hoz Siegler, Lan Gates, Qingye Lu. 2D and 3D metal-organic framework at the oil/water interface: A case study of copper benzenedicarboxylate. Advanced Materials Interfaces 6, 1801139 (2019)

24. Bo Xiao, Q., Yuan, R.A., Williams: Exceptional function of nanoporous metal organic framework particles in emulsion stabilisation. Chem. Commun. 49, 8208-8210 (2013)

25. Zhang, F., Liu, L., Tan, X., Sang, X., Zhang, J., Liu, C., Zhang, B., Han, B.: Guanying Yang. Pickering emulsions stabilized by a metal-organic framework (MOF) and graphene oxide (GO) for producing MOF/GO composites. Soft Matter 13, 7365-7370 (2017)

26. Reza Taheri, B., Kosasih, H., Zhu, A., Tieu: Dispersion stability and lubrication performance correlation of vegetable oil-In-water emulsions with nanoparticle-shielded oil droplets. Lubricants 6, 55 (2018)

27. He Liang, D., Guo, L., Ma, J., Luo: Investigation of film formation mechanism of oil-in-water (O/W) emulsions at high speeds. Tribol. Int. 109, 428-434 (2017)

28. Kumar, D., Daniel, J., Biswas, S.K.: Tribology of steel/steel interaction in oil-in-water emulsion; a rationale for lubricity. J. Colloid Interface Sci. 345, 307-315 (2010)

\section{Figures}




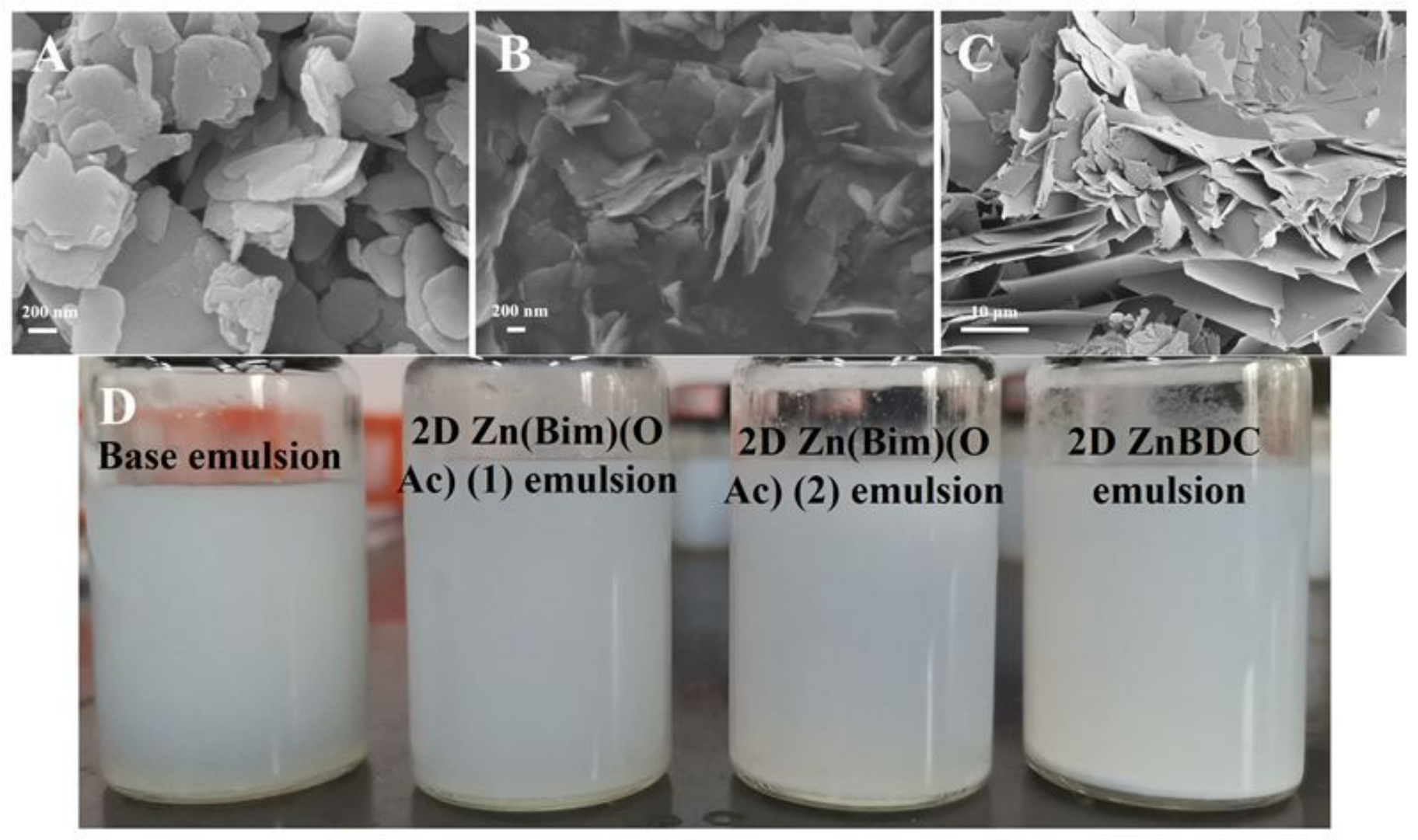

Figure 1

SEM images of (A) 2D Zn (Bim) (OAc) (1) nanosheets, (B) 2D Zn (Bim) (OAc) (1) nanosheets and (C) 2D ZnBDC nanosheets. (D) 2D Zn-MOFs digital image of oil in water emulsion. 


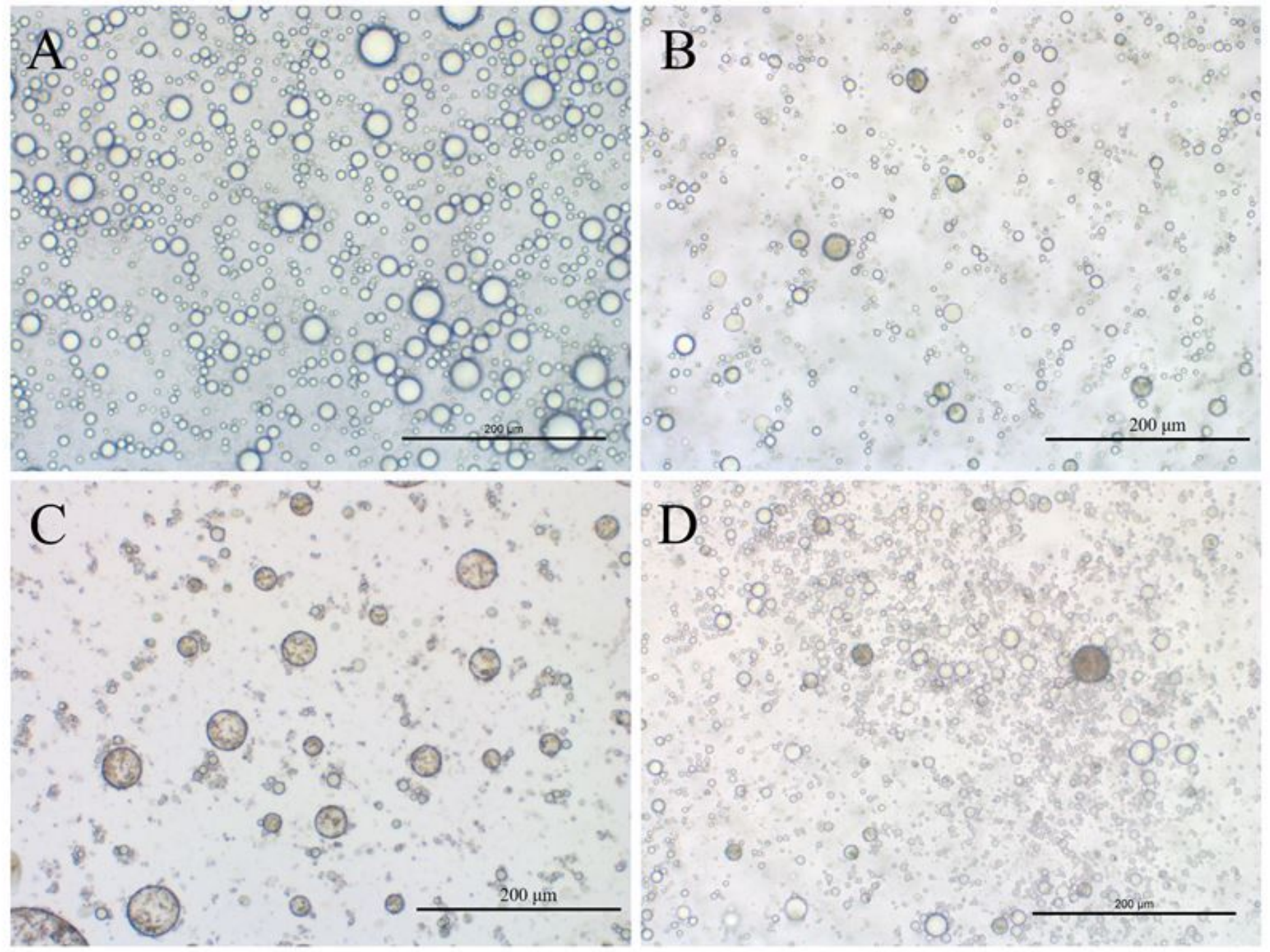

Figure 2

The droplet micrographs of base emulsion (A), 2D Zn (Bim) (OAc) (1) emulsion (B), 2D Zn (Bim) (OAc) (2) emulsion (C) and 2D ZnBDC emulsion(D). 

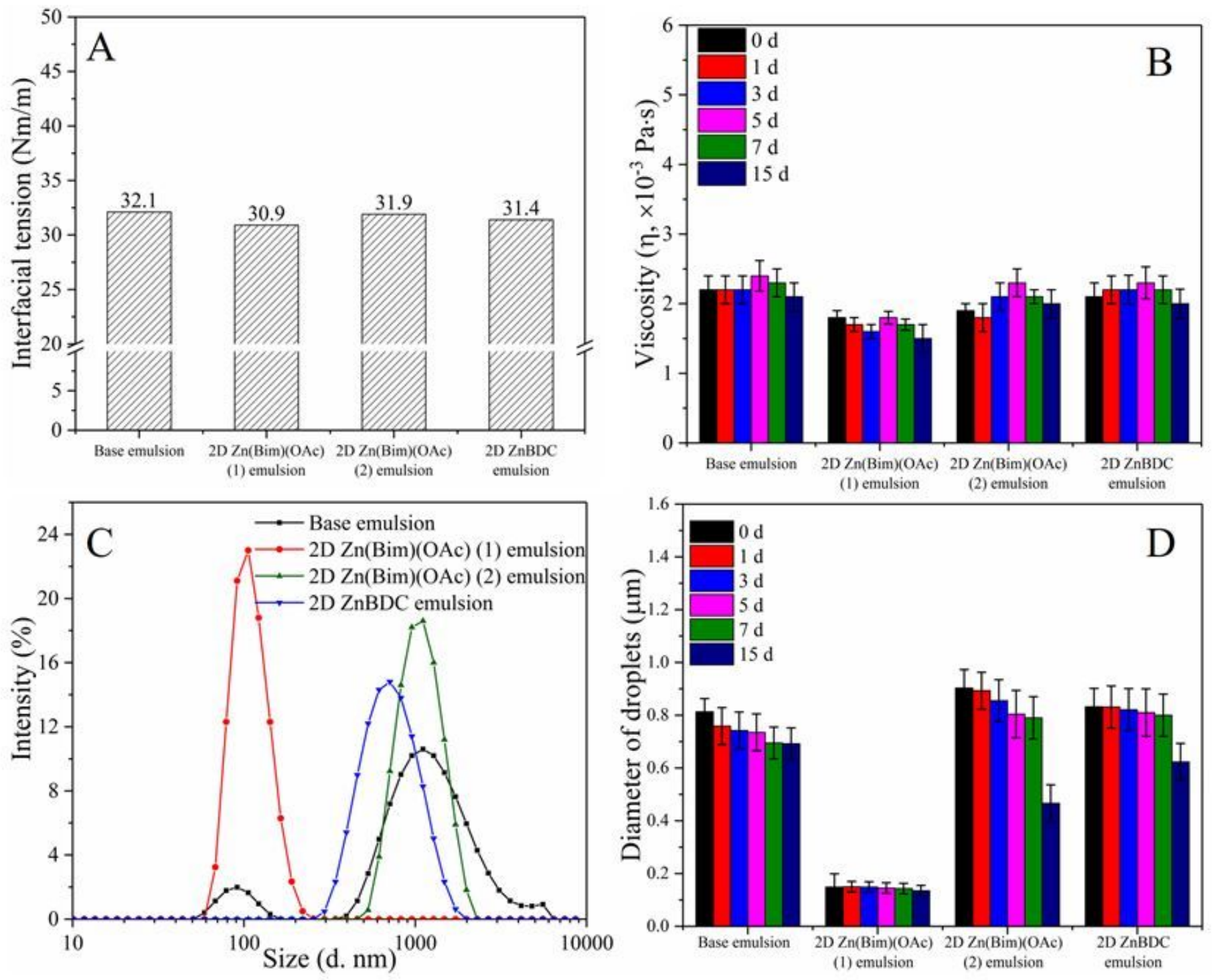

Figure 3

the interfacial tension (A) of base emulsion and 2D Zn-MOF emulsion, the change of viscosity with time (B), the droplet size distribution curve of emulsion oil (C) on 0 days and the change chart of droplet size with time. 

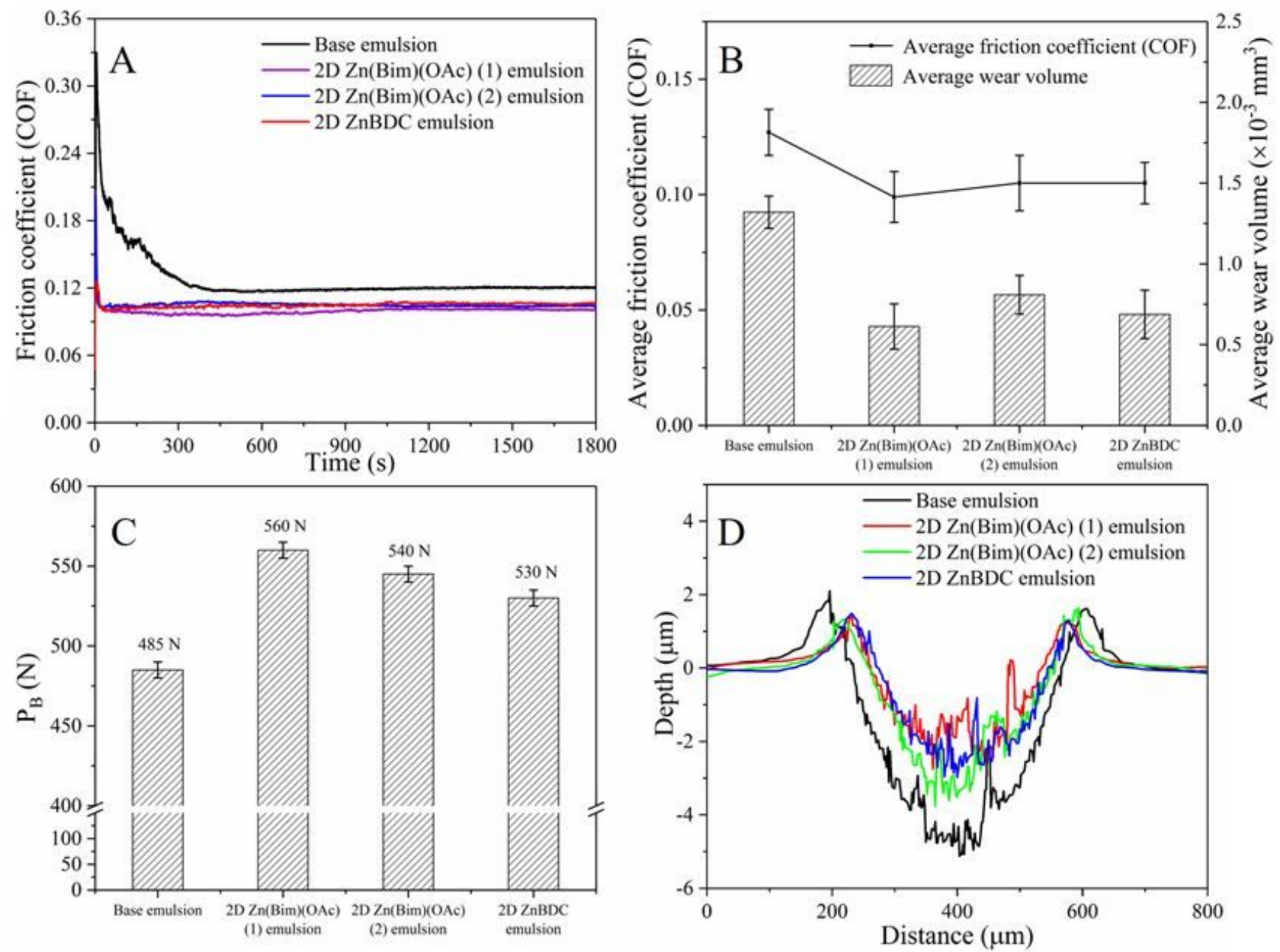

Figure 4

The friction coefficient curves of base emulsion and 2D Zn-MOFs emulsion at $30 \mathrm{~N}$ load and $2 \mathrm{~Hz}$ frequency (A), the average friction coefficient and average wear volume contrast diagram (B), extreme pressure (PB) comparison diagram (C) and slider wear trace curve (D). 

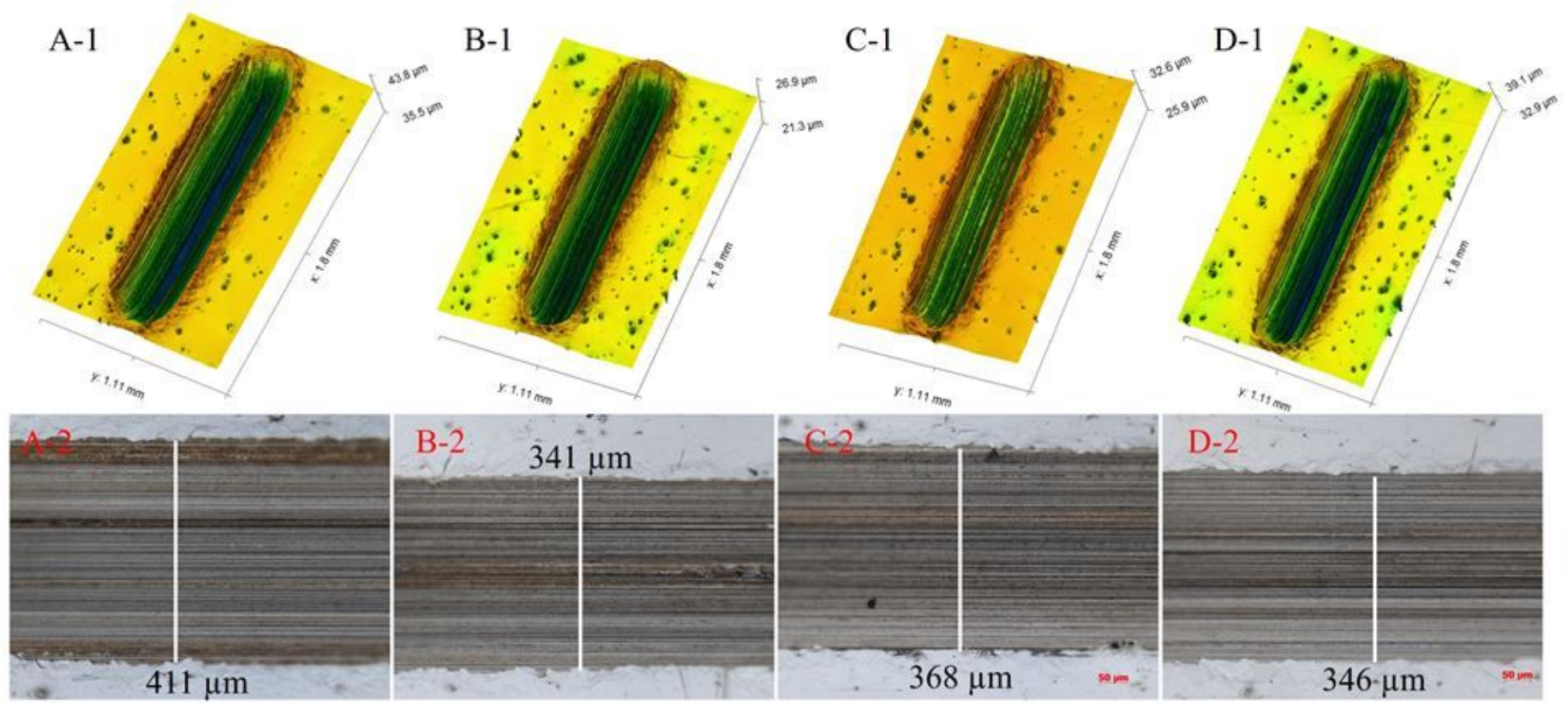

\section{Figure 5}

3D topographic map of wear marks and partial enlargement of partial wear scars: base emulsion (A-1, A2), 2D Zn (Bim) (OAC) (1) emulsion (B-1, B-2), 2D Zn (Bim) (OAC) (2) emulsion (C-1, C-2), and 2D ZnBDC emulsion (D-1, D-2). 

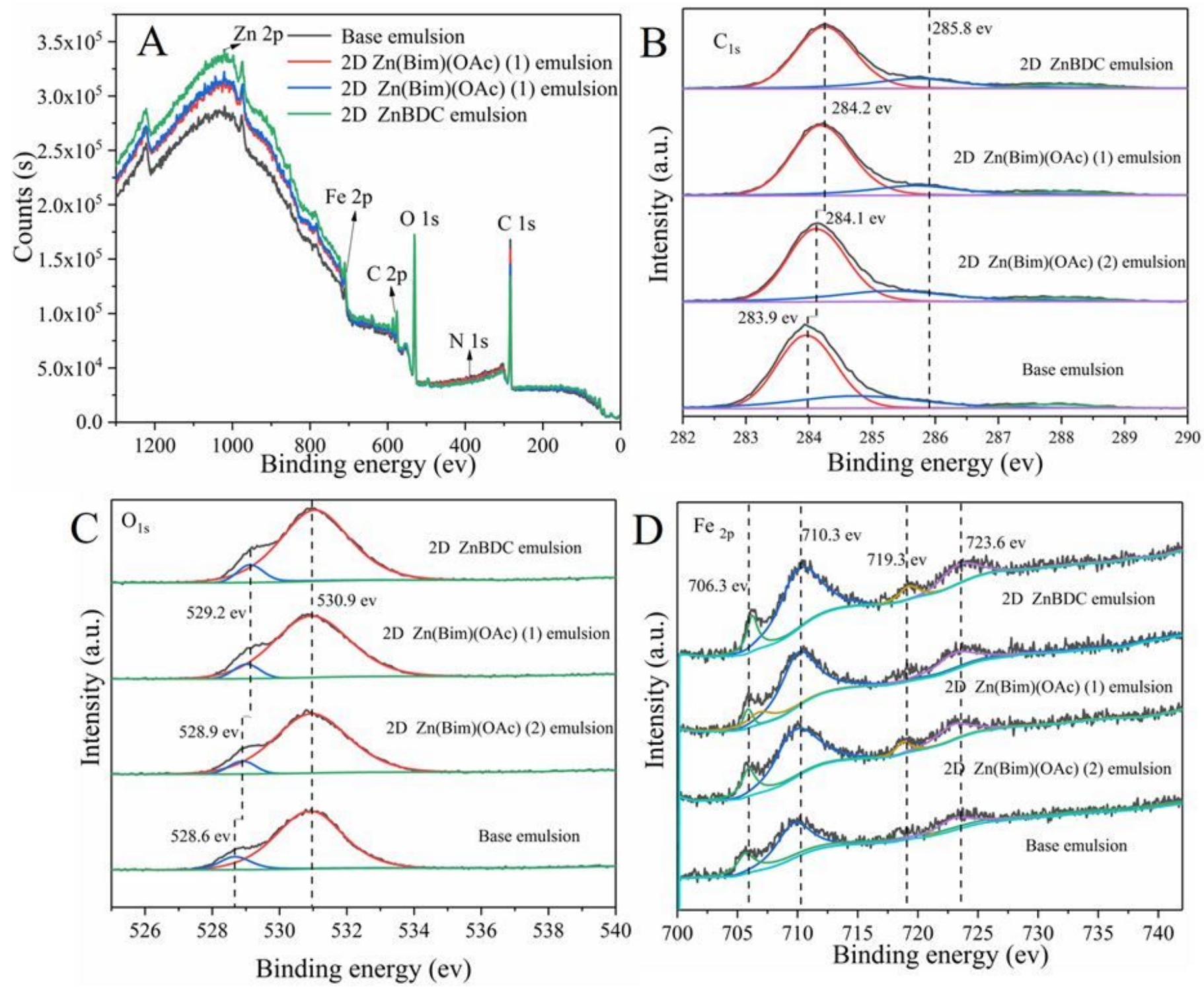

Figure 6

XPS spectrum of worn surface after friction test of base emulsion and 2D Zn-MOFs emulsion: (A) full spectrum, (B) C1S spectrum, (C) 01S spectrum and (D) Fe2p spectrum.

\section{Supplementary Files}

This is a list of supplementary files associated with this preprint. Click to download.

- GraphicalAbstract.jpg 